\title{
Microscopic polyangiitis presented with biopsy-confirmed pleuritis
}

\author{
Naoto Ishimaru1, Hisashi Ohnishi², Mao Fujiii2, Masako Yumura², Sho Yoshimura², \\ Saori Kinami ${ }^{1}$
}

1 Department of General Internal Medicine, Akashi Medical Center, Akashi, Hyogo
2 Department of Respiratory Medicine, Akashi Medical Center, Akashi, Hyogo Japan

\begin{abstract}
We describe a case of microscopic polyangiitis manifested as pleuritis confirmed by thoracoscopic biopsy. An 80-year-old man presented with a three-day history of shortness of breath and cough. Chest radiography revealed patchy opacities in the lower fields of the bilateral lung and right-sided pleural effusion. Thoracentesis revealed lymphocytic pleural exudates. Thoracoscopic biopsy specimens were compatible with fibrotic pleuritis. He developed rapidly progressive glomerulonephritis with elevated myeloperoxidase anti-neutrophil cytoplasmic antibody titer in blood and pleural effusion. Although the patient was resistant to two weekly courses of pulse steroid therapy, he was successfully treated with a five-day course of intravenous immunoglobulin.
\end{abstract}

\section{Introduction}

Microscopic polyangiitis (MPA) is classified as a necrotizing smallsized-vessel vasculitis that primarily affects the kidneys and lungs [1]. MPA has a fatality rate of $20-54 \%$ at five years after diagnosis and causes substantial long-term morbidity in survivors [2]. Common lung

Corresponding author: Naoto Ishimaru, Akashi Medical Center, 743-33, Ohkubo-cho Yagi, Akashi, Hyogo 674-0063, Japan.

Tel. +81.78.9361101 - Fax +81.78.9367456. E-mail: maru-tkb@umin.ac.jp

Key words: Microscopic polyangiitis; pleuritis; thoracoscopy.

Contributions: NI, manuscript writing; HO, MF, MY, SY, SY, review, inclusion of data according to single specialties and contribution to the case(s). All authors reviewed the manuscript and approved it.

Conflict of interest: The authors declare that they have no competing interests.

Acknowledgements: We thank Shenli Hew from Akashi Medical Center, for proofreading and editing of the manuscript.

Received for publication: 8 November 2017

Accepted for publication: 28 May 2018

(C) Copyright N. Ishimaru et al., 2018

Tipografia PI-ME Editrice, Italy

Monaldi Archives for Chest Disease 2018; 88:897

doi: 10.4081/monaldi.2018.897

This article is distributed under the terms of the Creative Commons Attribution Noncommercial License (by-nc 4.0) which permits any noncommercial use, distribution, and reproduction in any medium, provided the original author(s) and source are credited. involvement of MPA includes alveolar hemorrhage [3], interstitial pneumonia [4] and pulmonary nodules and/or masses (single or multiple) that can cavitate [5]. The manifestations of MPA presented with pleuritis are rare [6-9]. In addition, no reports have described thoracoscopic evaluation in patients with MPA accompanied by pleuritis. We report a case of MPA manifested as pleuritis confirmed by thoracoscopic biopsy.

\section{Case Report}

An 80-year-old man presented to a hospital with a three-day history of shortness of breath and cough. Chest radiography revealed patchy opacities in the lower fields of the bilateral lung and right-sided pleural effusion. He developed high fever on admission and was diagnosed with pneumonia. Intravenous sulbactam/ampicillin ( $4.5 \mathrm{~g} /$ day) followed by flomoxef ( $3 \mathrm{~g} /$ day) were administered. On day six, however, his symptoms worsened, and an increased pleural effusion was observed from a follow-up chest X-ray even after discontinuing all medications except for antibiotics. Antibiotics were changed to tazobactam/ piperacillin (13.5 g/day). On day 16, without any relief of his symptom and bilateral pleural effusion, he was referred to our hospital.

At 69 years old, he was diagnosed with hypertension and ischemic stroke. At 73 years old, he was diagnosed with epilepsy and bladder cancer, for which he underwent a transurethral resection of bladder tumor. He was prescribed with diazepam (6 mg/day), carbamazepine ( $800 \mathrm{mg} /$ day), phenobarbital ( $30 \mathrm{mg} /$ day), clobazam ( $5 \mathrm{mg} /$ day), lamotrigine $(25 \mathrm{mg} /$ day $)$, aspirin dialuminate $(81 \mathrm{mg} /$ day $)$, amlodipine besilate (5 mg/day), etizolam ( $0.5 \mathrm{mg} /$ day) and flunitrazepam ( $1 \mathrm{mg} /$ day). He has a history of smoking (40 sticks per day) and was a plumber for over 50 years where he might have been frequently exposed to asbestos. He had a weight of $46.4 \mathrm{~kg}$, height of $154.1 \mathrm{~cm}$ with a body mass index of 19.5. His temperature was $36.2{ }^{\circ} \mathrm{C}$, heart rate was 81 beats/min, blood pressure was $127 / 57 \mathrm{mmHg}$, respiratory rate was 16 breaths/min and oxygen saturation was $99 \%$ while breathing $1 \mathrm{~L} / \mathrm{min}$ of oxygen via a nasal cannula. Cardiac examination showed normal S1 and S2 sounds. Late-inspiratory fine crackles were heard in the lower fields of both the lungs. The abdomen was soft and non-tender. There was no skin lesion. Mild pitting edema was observed from the knees to the feet. The results of a neurologic examination were unremarkable. Laboratory findings obtained on admission are shown in Table 1. The patient had normocytic anemia with a hemoglobin level of $9.9 \mathrm{~g} / \mathrm{dL}$ and a mean corpuscular volume $100 \mathrm{fL}$. White blood cell count was slightly elevated at 10,780 cells $/ \mathrm{mm}^{3}$ with $78.1 \%$ neutrophils. C-reactive protein level was markedly elevated to $17.1 \mathrm{mg} / \mathrm{dL}$. Urinalysis revealed a red blood cell count of 30-49/high-power field and mild proteinuria. Chest radiography on admission revealed right-sided pleural effusion and patchy opacities in the lower fields of the left lung (Figure 1). Computed tomography (CT) of the chest revealed ground-glass opacity, interlobular septal thickening and traction bronchiectasis at both the lower lobes with pleural effusion more predominant in the right lung 
than the left lung (Figure 2). Sputum smear with Gram staining and culture yielded no significant microbes. Thoracentesis revealed that the effusion had a yellow cloudy appearance with exudative features (pH 7.5; specific gravity 1.033; total protein $3.5 \mathrm{~g} / \mathrm{dL}$; glucose $116 \mathrm{mg} / \mathrm{dL}$; lactate dehydrogenase $274 \mathrm{IU} / \mathrm{L}$; adenosine deaminase $32 \mathrm{IU} / \mathrm{L}$ ) and was rich in lymphocytes. No bacteria, fungi, or acid-fast bacilli were cultured, obviating the possibility of infection. Two hundred mL of fluid was removed.

Table 1. Laboratory data on admission.

\begin{tabular}{|c|c|c|c|}
\hline \multicolumn{2}{|c|}{ Complete blood count } & \multicolumn{2}{|c|}{ Blood chemistry } \\
\hline White blood cell & $10,780 / \mu \mathrm{L}$ & Total protein & $5.8 \mathrm{~g} / \mathrm{dL}$ \\
\hline Neutrophils & $78 \%$ & Alb & $1.8 \mathrm{~g} / \mathrm{dL}$ \\
\hline Lymphocytes & $8 \%$ & AST & $21 \mathrm{IU} / \mathrm{L}$ \\
\hline Basophils & $1 \%$ & ALT & $14 \mathrm{IU} / \mathrm{L}$ \\
\hline Eosinophils & $10 \%$ & LDH & $189 \mathrm{IU} / \mathrm{L}$ \\
\hline Monocytes & $4 \%$ & $\mathrm{Na}$ & $145 \mathrm{mEq} / \mathrm{L}$ \\
\hline Hemoglobin & $9.9 \mathrm{~g} / \mathrm{dL}$ & K & $3.5 \mathrm{mEq} / \mathrm{L}$ \\
\hline Platelet & $36.9 \times 10^{4} / \mu \mathrm{L}$ & BUN & 15 mg/dL \\
\hline Atrial blood gas & & Cre & $1.23 \mathrm{mg} / \mathrm{dL}$ \\
\hline $\mathrm{pH}$ & 7.408 & CRP & $17.1 \mathrm{mg} / \mathrm{dL}$ \\
\hline $\mathrm{PaO}_{2}$ & 81.6 torr & $\operatorname{Ig} \mathrm{G}$ & $1985 \mathrm{mg} / \mathrm{dL}$ \\
\hline $\mathrm{PaCO}_{2}$ & 41.0 torr & IgM & $85 \mathrm{mg} / \mathrm{dL}$ \\
\hline $\mathrm{HCO}_{3}^{-}$ & 25.3 torr & $\operatorname{IgA}$ & $611 \mathrm{mg} / \mathrm{dL}$ \\
\hline Urine analysis & & ANA & 1:40 \\
\hline Proteinuria & \pm & C3 & $100 \mathrm{mg} / \mathrm{dL}$ \\
\hline Occult blood & $3+$ & $\mathrm{C} 4$ & $21 \mathrm{mg} / \mathrm{dL}$ \\
\hline Glycosuria & - & $\mathrm{CH} 50$ & $56.3 \mathrm{U} / \mathrm{mL}$ \\
\hline Cast & RBC 30-49/HPF & $\mathrm{RF}$ & $19 \mathrm{IU} / \mathrm{mL}$ \\
\hline White blood cell & $<1 / \mathrm{HPF}$ & MPO-ANCA & $680 \mathrm{U} / \mathrm{mL}$ \\
\hline & & PR3-ANCA & $10 \mathrm{U} / \mathrm{mL}$ \\
\hline
\end{tabular}

WBC, white blood cell; HPF, high-power field.

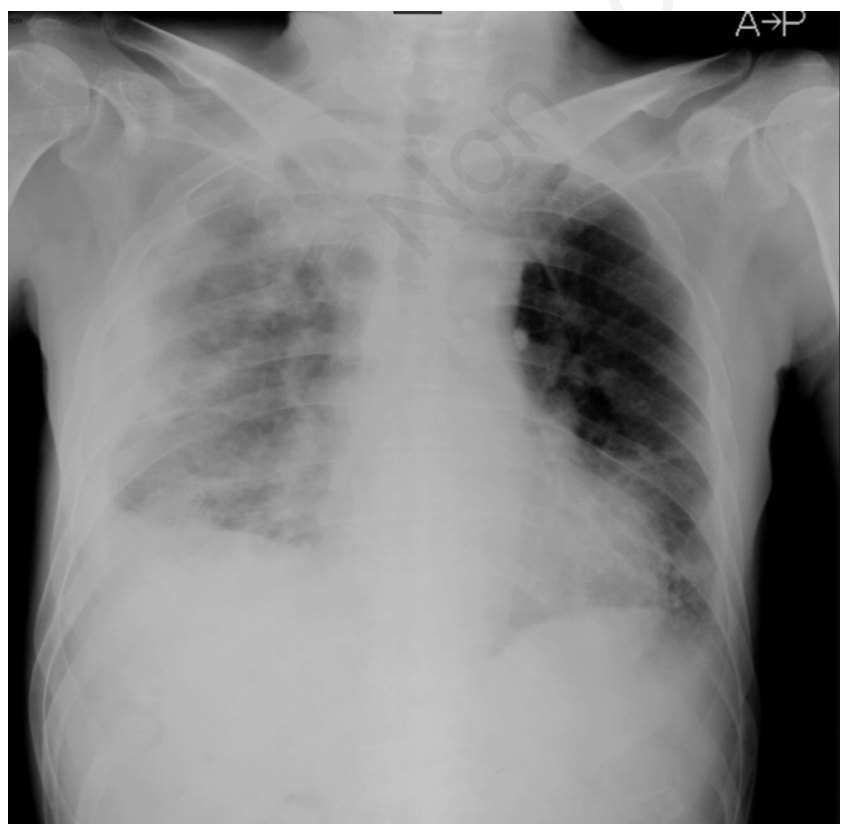

Figure 1. Chest radiography revealing right-sided pleural effusion and patchy opacities in the lower fields of the left lung.
To investigate the possibility of tuberculous pleural effusion and benign asbestos pleuritis malignant pleural mesothelioma, pleural biopsies were taken from the right parietal pleura using medical thoracoscopy. Thoracoscopic evaluation revealed inflamed and white, thickened appearance of the parietal and visceral pleuras with marked fibrinous network. Biopsy specimens demonstrated fibrotic change of the pleura with fibrin formation, compatible with fibrotic pleuritis (Figure 3). Although thoracentesis was performed, pleural effusion recurred. There was an elevation of serum creatinine level $(4.42 \mathrm{mg} / \mathrm{dL})$ with the onset of proteinuria ( $3.3 \mathrm{~g} /$ day) on hospital day 34 . Myeloperoxidase anti-neutrophil cytoplasmic antibody (MPO-ANCA) was markedly elevated $(680 \mathrm{U} / \mathrm{mL})$, whereas proteinase-3 (PR-3)-ANCA, rheumatoid factor (RF) and antinuclear antibody (ANA) were within the normal limit $(<10 \mathrm{U} / \mathrm{mL})$. The level of MPO-ANCA in the pleural effusion was also elevated $(1080 \mathrm{U} / \mathrm{mL})$. Taken together with interstitial change of the lungs, the findings were compatible with those of rapidly progressive glomerulonephritis (RPGN) caused by MPA. His renal function and urinary findings deteriorated in spite of the two weekly courses of pulse steroid therapy with methylprednisolone at $500 \mathrm{mg} /$ day for three days. We suspended the use of cyclophosphamide therapy because of the main concern for opportunistic infection. Therefore, we administered intravenous immunoglobulin (IVIg) (400 mg/kg/day) for five days, which resulted in decreased levels of both renal function and CRP, MPO-ANCA with a resolution of pleural fluid. The level of MPO-ANCA in

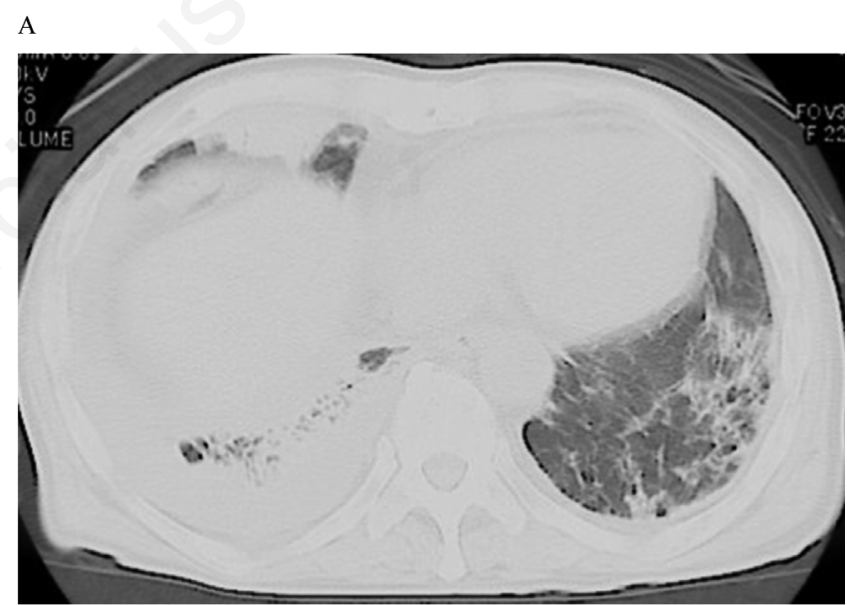

B

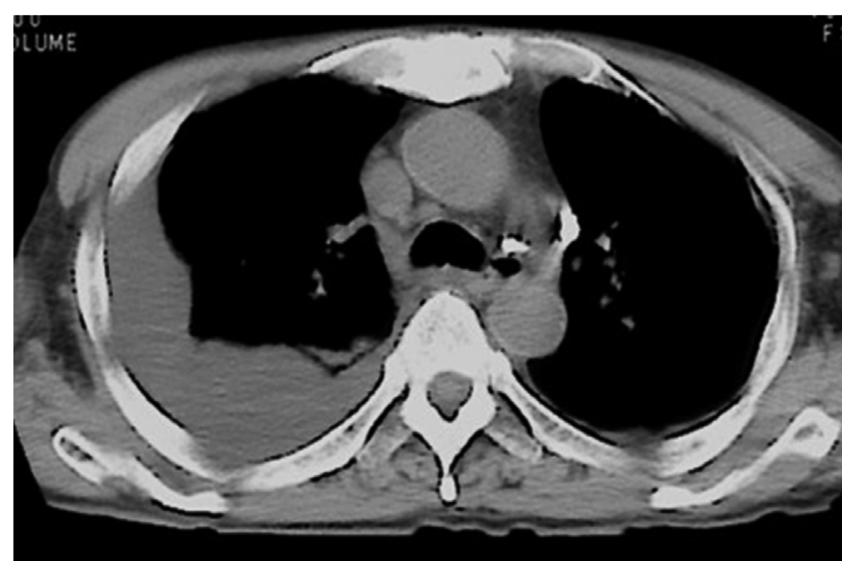

Figure 2. A) Computed tomography of the chest revealing groundglass opacity, interlobular septal thickening and traction bronchiectasis at both the lower lobes. B) Pleural effusion was more predominant in the right lung than the left lung. 
the pleural effusion was also decreased $(22 \mathrm{U} / \mathrm{mL})$. After prednisolone was tapered, we started azathioprine at $25 \mathrm{mg} /$ day. However, as the patient developed agranulocytosis, azathioprine was discontinued. We continued with only prednisolone to maintain the remission of the disease. The patient was discharged without any major complications except renal insufficiency. The patient's clinical course is summarized in Figure 4 .

\section{Discussion}

In this case report, we described a case of MPA manifested as pleuritis confirmed by thoracoscopic biopsy. The present case was finally classified as definite MPA based on the diagnostic criteria proposed by

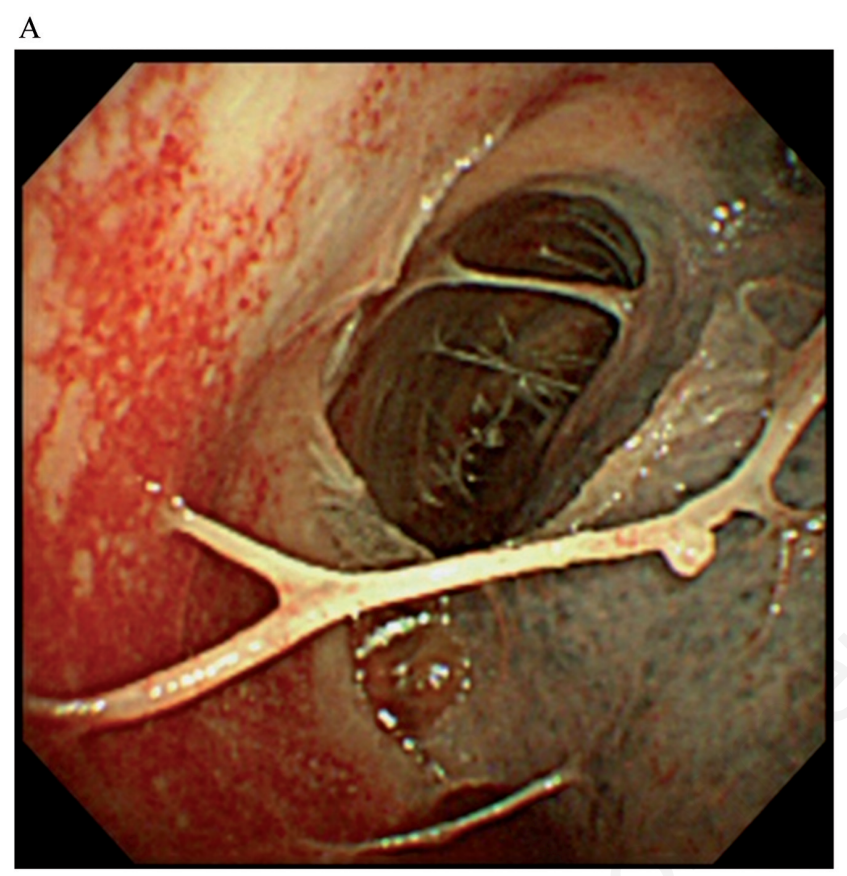

B

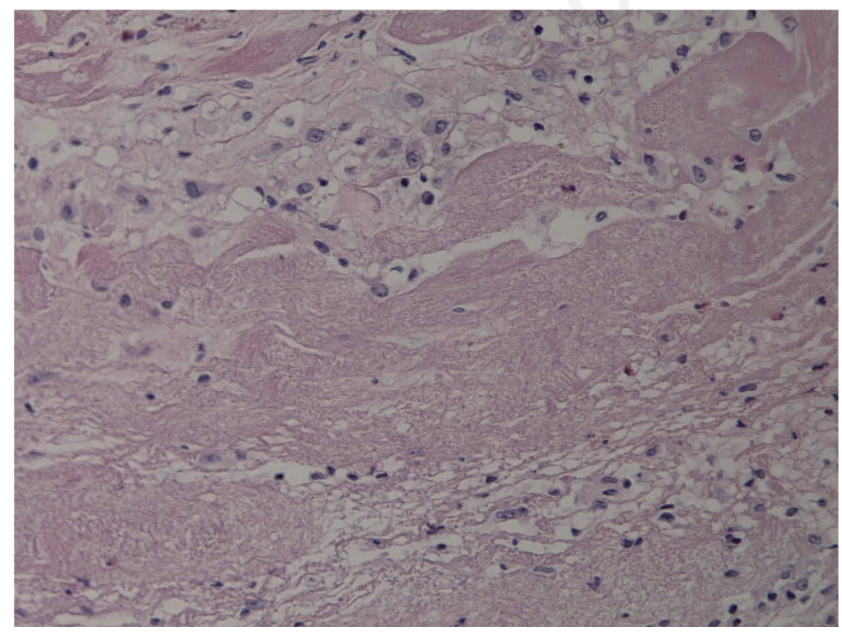

Figure 3. A) Thoracoscopic evaluation revealed inflamed and white, thickened appearance of the parietal and visceral pleuras with marked fibrinous network. B) Biopsy specimens of the right parietal pleura demonstrated fibrotic change of the pleura with fibrin formation, compatible with fibrotic pleurisy (Hematoxylin and eosin staining). the Japanese Ministry of Health and Welfare [10], that is, the presence of organ manifestations of the kidneys and lungs, and high serum levels of MPO-ANCA. Among the organ manifestation of MPA, kidney involvement is identified in 78-100\% of MPA cases, whereas lung involvement occurs in 7-61\% of MPA cases [6-9]. Both organs are affected in 7-59\% of MPA cases [6,7].

According to a report from the French Vasculitis Study Group, in which 85 patients with MPA were evaluated, only two (5.9\%) developed pleuritis [9]. Gaudin et al. reviewed 19 cases with MPA, in which only one patient had fibrinous pleuritis accompanied by interstitial fibrosis [11]. These reviews lack a precise description of pleural effusion. Nevertheless in rare cases, pleuritis has been reported as the only pulmonary manifestation in some cases of MPA [12,13], where exudates with a predominance of lymphocytes were confirmed by pleural fluid analysis. A report showed that the level of MPO-ANCA in pleural effusion was markedly elevated as that in serum [12]. Pleuritis antedated the appearance of RPGN in the report. In the present case, lymphocytic pleural exudates also presented with a high titer of MPO-ANCA which preceded the appearance of RPGN. Although no report has validated the utility of the measurement of MPO-ANCA in pleural effusion in cases of suspected MPA, a higher titer of MPO-ANCA in pleural effusion might suggest the predominant inflammation on the pleural surface, which was demonstrated in the thoracoscopic evaluation in our case. The present case revealed thoracoscopic findings which were not previously reported. Thoracoscopic evaluation is useful in the assessment of unexplained pleural exudates as it has an excellent diagnostic sensitivity and specificity (90-95\% and 100\%, respectively) [14]. To date, only one case report of MPA with pleuritis has described a closed pleural biopsy finding, which yielded the pathological diagnosis of nonspecific pleuritis [12]. Our pathological findings were compatible with the diagnosis.

Nonspecific pleuritis entails several possible alternative explanations. No vasculitis was found in the pleura. However, we diagnosed MPA-related pleuritis based on the elevated MPO-ANCA titer in pleural effusion. The resolution of pleural effusion was in accordance with the decrease in serum MPO-ANCA titer during the course of treatment for MPA. A report showed that $92 \%$ of patients with a nonspecific pleuritis

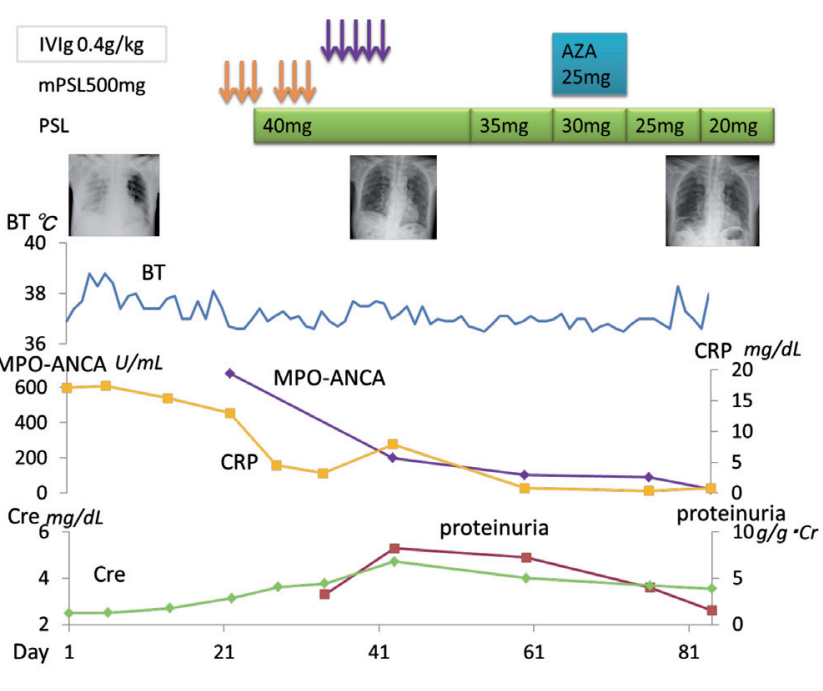

Figure 4. Clinical course of the patient. IVIg, intravenous immunoglobulin; MPSL, ethylprednisolone; PSL, prednisolone; AZA, zathioprine; MPO-ANCA; myeloperoxidase anti-neutrophil cytoplasmic antibody 
after thoracoscopy followed a benign course [15]. Hence, it is important to exclude malignancy such as malignant mesothelioma, with thoracoscopic visualization potentially playing a role in the diagnosis.

In spite of deteriorating kidney function after pulse steroid therapy, we successfully treated the patient with IVIg. A randomized study suggested that azathioprine or pulse cyclophosphamide therapy was effective for treating corticosteroid-resistant disease [16]. However, repeated cycles of immunosuppressive therapy are associated with bone marrow suppression, myelodysplasia and infection, especially among the elderly as in the present case. IVIg has been shown to be effective in the treatment of relapses of MPA in a multicenter prospective openlabel trial [17]. In addition to the immunosuppressive effects of glucocorticoids [18], IVIg provide immunoregulatory effects involving blockage of receptors for the Fc part of the IgG molecule (FCR) on macrophages and effector cells, attenuation of complement-mediated damage, control of autoreactive B cells and neutralization of active autoantibodies [19]. These effects may reflect the pathogenesis of MPA where ANCA interacts with FCR which are present in the neutrophils. On a similar note the alternative pathway of complement activation, TCell and B-cell are activated in MPA [20].

In unexplained pleuritis, MPA must be considered even before the development of RPGN. Especially in cases with lymphocytic pleural exudates, thoracoscopic biopsy is useful as a diagnostic aid. Early diagnosis, including MPO-ANCA testing, prompts appropriate treatment before the occurrence of serious and potentially life-threatening complications.

\section{References}

1. Jennette JC, Falk RJ, Andrassy K, et al. Nomenclature of systemic vasculitides. Proposal of an international consensus conference. Arthritis Rheum 1994;37:187-92.

2. Corral-Gudino L, Borao-Cengotita-Bengoa M, Del Pino-Montes J, Lerma-Marquez JL. Overall survival, renal survival and relapse in patients with microscopic polyangiitis: a systematic review of current evidence. Rheumatology (Oxford) 2011;50:1414-23.

3. Lauque D, Cadranel J, Lazor R, et al. Microscopic polyangiitis with alveolar hemorrhage. A study of 29 cases and review of the literature. Groupe d'Etudes et de Recherche sur les Maladies "Orphelines" Pulmonaires (GERM"0"P). Medicine (Baltimore) 2000;79:222-33.

4. Harper L, Morgan MD, Walsh M, et al. Pulse versus daily oral cyclophosphamide for induction of remission in ANCA-associated vasculitis: long-term follow-up. Ann Rheum Dis 2012;71:955-60.

5. Martinez F, Chung JH, Digumarthy SR, et al. Common and uncommon manifestations of Wegener granulomatosis at chest CT: radiologic-pathologic correlation. Radiographics 2012;32:51-69.
6. Arimura Y, Minoshima S, Tanaka U, et al. [Pulmonary involvement in patients with myeloperoxidase specific-antineutrophil cytoplasmic antibody]. Ryumachi 1995;35:46-55. [Article in Japanese].

7. Arulkumaran N, Periselneris N, Gaskin G, et al. Interstitial lung disease and ANCA-associated vasculitis: a retrospective observational cohort study. Rheumatology (Oxford) 2011;50:2035-43.

8. DeRemee RA, Homburger HA, Specks U. Lesions of the respiratory tract associated with the finding of anti-neutrophil cytoplasmic autoantibodies with a perinuclear staining pattern. Mayo Clin Proc 1994;69:819-24.

9. Guillevin L, Durand-Gasselin B, Cevallos R, et al. Microscopic polyangiitis: clinical and laboratory findings in eighty-five patients. Arthritis Rheum 1999;42:421-30.

10. Nakabayashi K, Hashimoto H. Microscopic polyangiitis. Tokyo: Ministry of Health, Labor, and Welfare of Japan; 2002; p. 24-6.

11. Gaudin PB, Askin FB, Falk RJ, Jennette JC. The pathologic spectrum of pulmonary lesions in patients with anti-neutrophil cytoplasmic autoantibodies specific for anti-proteinase 3 and antimyeloperoxidase. Am J Clin Pathol 1995;104:7-16.

12. Katae M, Takahashi K, Noto K, et al. [Microscopic polyangitis with pleuritis as the only pulmonary complication]. Nihon Kokyuki Gakkai Zasshi 2000;38:217-22. [Article in Japanese].

13. Shibuya H, Sano H, Osamura K, Kujime K, Hara K, Hisada T. Microscopic polyangiitis accompanied by pleuritis as the only pulmonary manifestation of occupational silica exposure. Intern Med 2010;49:925-9.

14. Janssen JP. Why you do or do not need thoracoscopy. Eur Respir Rev 2010;19:213-6.

15. Venekamp LN, Velkeniers B, Noppen M. Does 'idiopathic pleuritis' exist? Natural history of non-specific pleuritis diagnosed after thoracoscopy. Respiration 2005;72:74-8.

16. Ribi C, Cohen P, Pagnoux C, et al. Treatment of polyarteritis nodosa and microscopic polyangiitis without poor-prognosis factors: A prospective randomized study of one hundred twenty-four patients. Arthritis Rheum 2010;62:1186-97.

17. Martinez V, Cohen P, Pagnoux C, et al. Intravenous immunoglobulins for relapses of systemic vasculitides associated with antineutrophil cytoplasmic autoantibodies: results of a multicenter, prospective, open-label study of twenty-two patients. Arthritis Rheum 2008;58:308-17.

18. Coutinho AE, Chapman KE. The anti-inflammatory and immunosuppressive effects of glucocorticoids, recent developments and mechanistic insights. Mol Cell Endocrinol 2011;335:2-13.

19. Kazatchkine MD, Kaveri SV. Immunomodulation of autoimmune and inflammatory diseases with intravenous immune globulin. N Engl J Med 2001;345:747-55.

20. Kallenberg CG, Stegeman CA, Abdulahad WH, Heeringa P. Pathogenesis of ANCA-associated vasculitis: new possibilities for intervention. Am J Kidney Dis 2013;62:1176-87. 